\title{
Comparison of methods for quantitative biomolecular interaction analysis
}

\author{
Monika Conrad $^{1}$ • Peter Fechner ${ }^{1}$ - Günther Proll ${ }^{1}$ - Günter Gauglitz ${ }^{1}$ \\ Received: 5 July 2021 / Revised: 11 August 2021 / Accepted: 16 August 2021 / Published online: 10 September 2021 \\ (C) The Author(s) 2021
}

\begin{abstract}
In order to perform good kinetic experiments, not only the experimental conditions have to be optimized, but the evaluation procedure as well. The focus of this work is the in-depth comparison of different approaches and algorithms to determine kinetic rate constants for biomolecular interaction analysis (BIA). The different algorithms are applied not only to flawless simulated data, but also to real-world measurements. We compare five mathematical approaches for the evaluation of binding curves following pseudo-first-order kinetics with different noise levels. In addition, reflectometric interference spectroscopy (RIfS) measurements of two antibodies are evaluated to determine their binding kinetics. The advantages and disadvantages of the individual approach will be investigated and discussed in detail. In summary, we will raise awareness on how to evaluate and judge results from BIA by using different approaches rather than having to rely on "black box" closed (commercial) software packages.
\end{abstract}

Keywords Reflectometric interference spectroscopy $\cdot$ Biomolecular interaction analysis $\cdot$ Binding kinetics $\cdot$ Association rate constant $\cdot$ Pseudo-first-order kinetics

\section{Introduction}

The analysis of biomolecular interaction is a fascinating field of research. Especially important is the analysis of binding kinetics, which allows the determination not only of the thermodynamic affinity constant, but also of the kinetic association and dissociation rate constants. The knowledge of these constants leads to a deeper understanding of how biological systems function at the molecular level, which can be very useful for pharmaceutical research and rational design of therapeutics [1].

Biomolecular interaction analysis (BIA) aims to quantify interaction patterns in order to describe events between biomolecules, e.g. antibody and its antigen. The analysis of binding events is error-prone because of the user influence on experimental design, on the used analytical method, on the quality of measurement, and on data evaluation. As one-to-one interaction is the

Published in the topical collection celebrating ABCs 20th Anniversary.

Monika Conrad

monika.conrad@uni-tuebingen.de

1 Institute of Physical and Theoretical Chemistry (IPTC), Eberhard Karls Universität Tübingen, Auf der Morgenstelle 18, 72076 Tübingen, Germany simplest model available, it is often applied. However, the experimental setup must be designed carefully to make sure the chosen mathematical model is applicable. Since it is a difficult task to achieve good experimental design, there are reviews pointing out that the quality of published biosensor work is often poor $[2,3]$. But there are also publications demonstrating how good kinetic analysis should be performed [4-6]. For example, a study with different Biacore users showed that it is possible to obtain reproducible kinetic constants with proper instructions [7].

The principle of BIA is to detect time-resolved specific interaction of an analyte in continuous flow with an immobilized ligand [8]. Many papers determine binding constants or rates of interactions in immunology $[9,10]$, drug screening $[11,12]$, or even proteomics research [13, 14]. Especially suitable for BIA are label-free techniques as they avoid disturbances from conjugated markers or complex handling of radioactive material. Common techniques to measure label-free binding are isothermal titration calorimetry (ITC) [15] or surface plasmon resonance (SPR) [16]. SPR belongs to the direct optical detection methods [17, 18] alongside integrated optical grating coupler [19] or reflectometric interference spectroscopy (RIfS) [20]. They allow time-resolved measurements yielding thermodynamic and kinetic information. However, as mentioned in a recent review [21] matrix, both instrumentation and flow influence measurements. 
Commercial software for data evaluation is available, e.g. from Biacore [22], TraceDrawer [23], or Scrubber [24]. Besides, there is open-source software available like Anabel [25] or EvilFit [26]. A problem when using available software is that it is irrelevant whether the user understands what exactly the software does, which might lead to wrong application and consequently to false constants. The most important question the user should ask himself is whether the assumed model is correct. This requires an understanding of biomolecular processes in the homogeneous phase, of transport processes to or from the surface, and of the kinetic processes at the biosensor [27]. RIfS belongs to the heterogeneous immunoassays; thus, the immune reaction takes place on a solid phase with an immobilized component and ideally only effects at the surface are monitored.

Kinetic analysis of binding curves is a long-established procedure. Often the complex binding process is reduced to the reaction between the immobilized ligand and the analyte in solution which represents one-to-one kinetics. This one-toone interaction can be described by pseudo-first-order kinetics, if the analyte flows over the surface resulting in its concentration remaining constant. Assuming one-to-one interaction, the rate and affinity constants can be easily calculated, if the association and dissociation curves are measured for various analyte concentrations. Experimental conditions should be adapted to avoid deviations from the pseudo-first-order kinetic model as described in [5]. Mass transport limitation, for example, can be reduced by using fast flow rates and by reducing the immobilization level of the recognition element. It can be verified by varying the flow rate [28].

For the calculation of the rate constants, different mathematical approaches can be used: linear transformation of the primary data by use of the derivative [9] or by use of the integral of the binding curve, or the integrated rate equation, which gives an exponential function [29]. There are several factors that can prevent the binding curve to follow pseudofirst-order kinetics: mass transfer [30], rebinding of analyte [31], bivalency or even other orders of analyte, two-state reaction [10], parallel reactions, or competing reactions [32]. Some of these effects can be ruled out by a careful experimental design, but it is also possible to apply more complex models, if numerical integration in combination with global data fitting is used [32, 33].

In this paper, we compare evaluation tools using simulated kinetic data and determine how they can cope with different noise levels. Deviations from pseudo-first-order reaction kinetics can be ruled out when using simulated data allowing the comparison of different evaluation approaches without being flawed by device-specific error sources. In order to mimic real measurement data, noise was added to the simulated data. The effect of evaluating only a part of the association phase in contrast to evaluating the entire association phase is examined. In addition, these evaluation tools are used with experimental data of two antibodies measured with RIfS for comparison in order to apply the results of the evaluation of simulated data. For real data, it is important to check whether the goal of avoiding mass transport by reducing the immobilization level of the recognition element is achieved. This paper should be a guideline for BIA evaluation, point out what to look out for experimentally, and help with interpretation and verification of results.

\section{Materials and methods}

\section{Materials}

Common chemicals were purchased from Sigma-Aldrich (Taufkirchen, Germany) or Fluka (Neu-Ulm, Germany). The monoclonal $\mathrm{IgG}$ antibodies to amitriptyline (host mouse) clone 202 and clone TU-11 were purchased from Aviva Systems Biology Corporation (San Diego, USA) and antikoerper-online.de (Aachen, Germany) respectively. Poly(ethylene glycol) diamine (PEG-DA, MW 2000 Da) and a-methoxy- $\omega$-amino PEG (PEG-MA, MW 2000 Da) were purchased from Rapp Polymere (Tübingen, Germany). Phosphate-buffered saline (PBS) consisted of $150 \mathrm{mM}$ sodium chloride and $10 \mathrm{mM}$ potassium phosphate at $\mathrm{pH}$ 7.4. The solution used for regeneration of the sensor surface was guanidine hydrochloride ( $\mathrm{GdnHCl}, 6 \mathrm{M}, \mathrm{pH} 1.5)$. RIfS glass transducers $(1 \mathrm{~cm} \times 1 \mathrm{~cm})$ consisting of a 1-mm glass substrate with a layer of $10 \mathrm{~nm} \mathrm{Ta} \mathrm{O}_{5}$ covered with $330 \mathrm{~nm} \mathrm{SiO}{ }_{2}$ on top were obtained from Schott AG (Mainz, Germany).

\section{Surface chemistry for RIfS transducers}

The RIfS experiments were performed as described in [20]. The ligand nortriptyline (NRT) was immobilized on glass transducers using amine-coupling chemistry based on [34] and similar to [35]. The transducers $(1 \times 1 \mathrm{~cm})$ were first cleaned for $30 \mathrm{~s}$ in $\mathrm{KOH}$ (6 M) solution, then washed with $\mathrm{H}_{2} \mathrm{O}$. Next, they were cleaned and activated for $15 \mathrm{~min}$ using freshly prepared piranha solution (3:2 conc. $\mathrm{H}_{2} \mathrm{SO}_{4}: \mathrm{H}_{2} \mathrm{O}_{2}(30 \%)$ ). After washing the transducers with $\mathrm{H}_{2} \mathrm{O}$ and drying under nitrogen, they were modified with 3-glycidyloxypropyl-trimethoxysilane (GOPTS) for $1 \mathrm{~h}$. The transducers were cleaned with acetone and dried under nitrogen. The polymer mixture of PEG-DA and PEG-MA (1:1000) for the kinetic analysis was bound covalently onto the GOPTS layer using $20 \mu \mathrm{l} \mathrm{PEG}(4 \mathrm{mg} / \mathrm{ml}$ in dichloromethane DCM). After reacting overnight at $70{ }^{\circ} \mathrm{C}$, the transducers were cleaned with $\mathrm{H}_{2} \mathrm{O}$ and dried under nitrogen. The amino functions of PEG-DA were transferred into carboxyl functions using $10 \mu \mathrm{l}$ of dissolved glutaric acid (GA) $(0.67 \mathrm{mg} / \mu \mathrm{l} \mathrm{GA}$ in DMF). Each transducer was covered with another transducer in a DMF vapour-saturated chamber for at least $6 \mathrm{~h}$. Afterwards, the transducers were cleaned with DMF and $\mathrm{H}_{2} \mathrm{O}$ and dried under 
nitrogen. Subsequently, NRT was immobilized on the sensor surface using $\mathrm{N}, \mathrm{N}^{\prime}$-diisopropyl-carbodiimide (DIC) and Nhydroxysuccinimide (NHS) as coupling reagents. NHS $(150 \mathrm{mg})$ and DIC $(302 \mu \mathrm{l})$ were dissolved in $1 \mathrm{ml} \mathrm{DMF}$, and the transducers covered with the solution in a DMF vapoursaturated chamber for $4 \mathrm{~h}$. After cleaning with DMF and acetone, and drying under nitrogen, the transducers were incubated with NRT $\left(2 \mathrm{mg} / \mathrm{ml}\right.$ in $\left.\mathrm{H}_{2} \mathrm{O}\right)$ in a water vapour-saturated chamber overnight. Then, the transducers were washed and dried under nitrogen.

\section{Measurement}

RIfS was chosen for the BIA experiments because we have the best experience with this system and it allows the most experimental modifications. It is based on interference of white light at thin films [36]. At phase boundaries, part of the light is transmitted and part is refracted. The reflected partial beams superimpose resulting in an interference spectrum described in detail in $[18$, 36]. A change in optical thickness (nd; product of refractive index and physical thickness), which might be caused by antibody binding to antigen immobilized on the surface, results in a shift of the interference spectrum. Monitoring the optical thickness over time allows time-resolved detection [20] and typical binding curves are obtained.

To collect binding data, measurements are performed similar to $[35,37,38]$. First, the transducer surface was flushed with buffer (baseline). Then, different concentrations of the analyte (33 to $500 \mathrm{nM}$ (antibody $150 \mathrm{kDa}$ )) in PBS pH 7.4 were injected at a flow rate of $0.5 \mu \mathrm{l} / \mathrm{s}$ at room temperature without temperature control. The dimensions of the flow cell are $50 \mu \mathrm{m}$ channel depth, $1 \mathrm{~mm}$ channel width, and $4 \mathrm{~mm}$ channel length. The complex was allowed to associate for $600 \mathrm{~s}$ and dissociate for $900 \mathrm{~s}$. The sample and buffer were separated by air to prevent diffusion decreasing the sample concentration towards the end of the association and low sample concentration in the buffer during dissociation. The surfaces were regenerated with a $400 \mathrm{~s}$ injection of $\mathrm{GdnHCl}$. Finally, another baseline was measured by flushing the cell with buffer again. Triplicate injections of each sample were flowed over the surface in random order.

\section{Simulation of kinetic data}

Data were simulated using the 1:1 (Langmuir) binding model of BIAevaluation 4.1.1 with $k_{\mathrm{a}}=1 \cdot 10^{4} \mathrm{M}^{-1} \mathrm{~s}^{-1}$ and $k_{\mathrm{d}}=1 \cdot 10^{-3} \mathrm{~s}^{-1}$. The maximum analyte binding capacity Rmax was set to $1 \mathrm{~nm}$, and the bulk refractive index contribution RI was set to 0 . The analyte concentrations for the simulations were $500 \mathrm{nM}, 333 \mathrm{nM}, 167 \mathrm{nM}, 133 \mathrm{nM}, 100 \mathrm{nM}$, $67 \mathrm{nM}$, and $33 \mathrm{nM}$. The association and dissociation times were set to $600 \mathrm{~s}$ and $900 \mathrm{~s}$, respectively. A value was taken every $5 \mathrm{~s}$ which is the same rate as in the RIfS measurements.
Noise was added by use of Matlab R2020b with an amplitude of $0.01 \mathrm{~nm}$ and $0.001 \mathrm{~nm}$ using random numbers with the following command where $y$ is the simulated data and $r$ the amplitude of noise:

$y$ Noisy $=y+2 \cdot r \cdot \operatorname{rand}($ length $(y), 1)-2 \cdot r \cdot 0.5$

The noise levels used were $0.001 \mathrm{~nm}$ and $0.01 \mathrm{~nm}$, which is $1 \%$ of Rmax and about $1 / 14$ of the smallest increase of optical thickness $(0.14 \mathrm{~nm}$ for $33 \mathrm{nM}) .0 .01 \mathrm{~nm}$ noise is very large in comparison with real measurement data where the noise is typically around $0.001 \mathrm{~nm}$ noise.

Furthermore, the residuals obtained by mono-exponential fit of one of the experimental binding curves $(67 \mathrm{nM}$ clone $202,25-525 \mathrm{~s}$ of the association phase) were added to the simulated curve of the same concentration.

\section{Data evaluation}

Data evaluation was performed for the simulated and the experimental data. To evaluate the measured data, the first five data points and the last 20 data points of the association were left out (shown in Fig. S1). The methods used for the evaluation of the association phase were mono-exponential fit, derivative, and integration. The dissociation phase was evaluated with an exponential decay function and a linearization method. In addition, the BIAevaluation software was used to evaluate both phases. In order to compare how well different methods calculate the true rate constants for simulated data, relative deviations were calculated by

$\frac{k_{\text {calculated }}-k_{\text {true }}}{k_{\text {true }}}$

\section{Association}

The association phase was evaluated using three different methods for three different areas of the association phase. For simulated data, first, the entire association phase was evaluated, then the first half, and finally, the association until an optical thickness of $0.5 \mathrm{~nm}$ was reached. For experimental data, the part of the association phase where the derivative showed linear behaviour was evaluated.

\section{Mono-exponential fit}

The data of the association phase were fitted with the MnMolecular formula provided by Origin Pro 2021 (iteration algorithm: Levenberg Marquardt) which is $y=A\left(1-e^{-k(x-x c)}\right)$. The theoretical integrated rate equation is

$\Gamma(t)=\Gamma_{\mathrm{eq}}\left(1-e^{-k_{\mathrm{obs}} \cdot t}\right)$ 
where $\Gamma(t)$ is the surface load capacity over time, $\Gamma_{\mathrm{eq}}$ the equilibrium surface load capacity, and $k_{\mathrm{obs}}$ the observed binding rate constant which also describes the curvature of the calculated fitting curve. In the case of RIfS, the measured signal is the difference in the optical thickness $\Delta$ nd.

\section{$k_{\text {obs }}$ linearization}

The calculated $k_{\mathrm{obs}}$ for different concentrations are related to the rate constants by $k_{\mathrm{obs}}=k_{\mathrm{a}} \cdot c+k_{\mathrm{d}}$ with the association rate constant $k_{\mathrm{a}}$ and the dissociation rate constant $k_{\mathrm{d}}$. By plotting $k_{\text {obs }}$ vs. the antibody concentration, the association rate constant is obtained by linear regression performed by Origin (instrumental error weighting $=1 / \mathrm{ei}^{\wedge} 2$ ) where $k_{\mathrm{a}}$ represents the slope and $k_{\mathrm{d}}$ represents the $y$-axis intercept of the linear fit. For simulated data, error bars of $k_{\mathrm{obs}}$ represent the standard error of the fit, while for measured data, $k_{\mathrm{obs}}$ values are calculated as the mean of the triplicate measurements where the error bars indicate the standard deviation of the mean.

\section{Derivative}

For the evaluation by derivative, the derivative of the surface load capacity $\mathrm{d} \Gamma(t) / \mathrm{d} t$ (in this case $\mathrm{d}(\Delta \mathrm{nd}) / \mathrm{d} t$ ) is plotted against the $\Gamma(t)$ (here $\Delta \mathrm{nd}$ ) where $k_{\mathrm{obs}}$ is obtained as the negative slope after linear regression.

$\frac{\mathrm{d} \Gamma(t)}{\mathrm{d} t}=k_{\mathrm{a}} \cdot c \cdot \Gamma_{\max }-k_{\mathrm{obs}} \cdot \Gamma(t)$

\section{Integration}

Integrating this rate equation gives

$\frac{\Gamma\left(t_{2}\right)-\Gamma\left(t_{1}\right)}{t_{2}-t_{1}}=k_{\mathrm{a}} \cdot c \cdot \Gamma_{\max }-k_{\mathrm{obs}} \cdot \frac{\int_{t_{1}}^{t_{2}} \Gamma(t) \mathrm{d} t}{t_{2}-t_{1}}$

When starting from the beginning with $t_{1}=0$ and $\Gamma(0)=0$, this equation becomes

$\frac{\Gamma(t)}{t}=k_{\mathrm{a}} \cdot c \cdot \Gamma_{\max }-k_{\mathrm{obs}} \cdot \frac{\int_{0}^{t} \Gamma(t) \mathrm{d} t}{t}$

The data of the association phase were integrated and divided by the integration time interval to obtain the $X$ data. The integration of $\Delta$ nd was performed with Origin (mathematical area). The difference between the optical thickness at the end of the integration and at the start of the integration divided by the time interval provided the $Y$ data. A plot of $\Gamma(t) / t$ vs. $\int_{0}^{t} \Gamma(t) \mathrm{d} t / t$ gives $k_{\text {obs }}$ as the negative slope. The integration can be performed in two directions starting from the beginning (Int f) or from the end of the association phase (Int b). In both cases, a linear plot of the data is achieved with deviations from linearity for the first values (Fig. 1). For simulated data with $0.001 \mathrm{~nm}$ noise, the first ten values after integration were masked, for simulated data with $0.01 \mathrm{~nm}$ noise and experimental data, the values to be masked were determined by assessing which part of the data showed a linear behaviour. The integration was also preformed starting where half of the surface load capacity at the end of the association phase was reached.

\section{Dissociation}

Data evaluation of the dissociation phase was performed with an exponential fit and a linearization method. The theoretical equation for dissociation is

$\Gamma(t)=\Gamma_{0} e^{-k_{\mathrm{d}} \cdot t}$

where $\Gamma_{0}$ is the surface load capacity at the beginning of the dissociation and $k_{\mathrm{d}}$ is the dissociation rate constant. The used function was ExpDecay1 in Origin $y=y_{0}+A_{1} e^{-\frac{x-x_{0}}{t_{1}}}$ where $k_{\mathrm{d}}$ can be calculated from $t_{1}$ by $k_{\mathrm{d}}=\frac{1}{t_{1}} \cdot y_{0}$ is the $y$-offset. Strict one-to-one interaction assumes that all bound analyte molecules can dissociate, but if the fitting function contains a $y$ offset, it allows analyte molecules to stay on the surface and thus describes back bonding.

For linearization, this function is transformed to

$\ln \left(\frac{\Gamma(0)}{\Gamma(t)}\right)=k_{\mathrm{d}} \cdot t$

A plot of $\ln \left(\Gamma_{0} / \Gamma(t)\right)$ vs. $t$ gives $k_{\mathrm{d}}$ as the slope.

\section{BIAevaluation}

For the evaluation with BIAevaluation, the beginning of the association phase was set to zero on the $x$ and $y$ scale (XTransform, Y-Transform). The association phase was evaluated from 100 to $500 \mathrm{~s}$ for simulated data and until an optical thickness of $0.5 \mathrm{~nm}$ for clone 202 and $0.6 \mathrm{~nm}$ for clone TU-11 was reached. The dissociation phase was evaluated from 700 to 1400 s using Fit:Kinetics Simultaneous ka/kd and Fit:Kinetics Separate ka/kd with the 1:1 (Langmuir) model. For simultaneous ka/kd, BIAevaluation performs a global fit of association and dissociation of all concentrations. The chosen settings for the parameters were constant concentration; global fit of ka, kd, and Rmax; and local fit of RI. For Separate $\mathrm{ka} / \mathrm{kd}$, the dissociation phase is evaluated first. Settings were global fit of $\mathrm{kd}$ with local fit of R0 and offset, t0 was set constant to $605 \mathrm{~s}$. Then, the obtained $\mathrm{kd}$ value is used as a constant for the global fit of ka of the association phase with constant concentrations and local fit of $\mathrm{t} 0$ and RI. For measured data, this setting for separate $\mathrm{ka} / \mathrm{kd}$ resulted in too many fitted parameters; thus, t0 was fitted globally. 

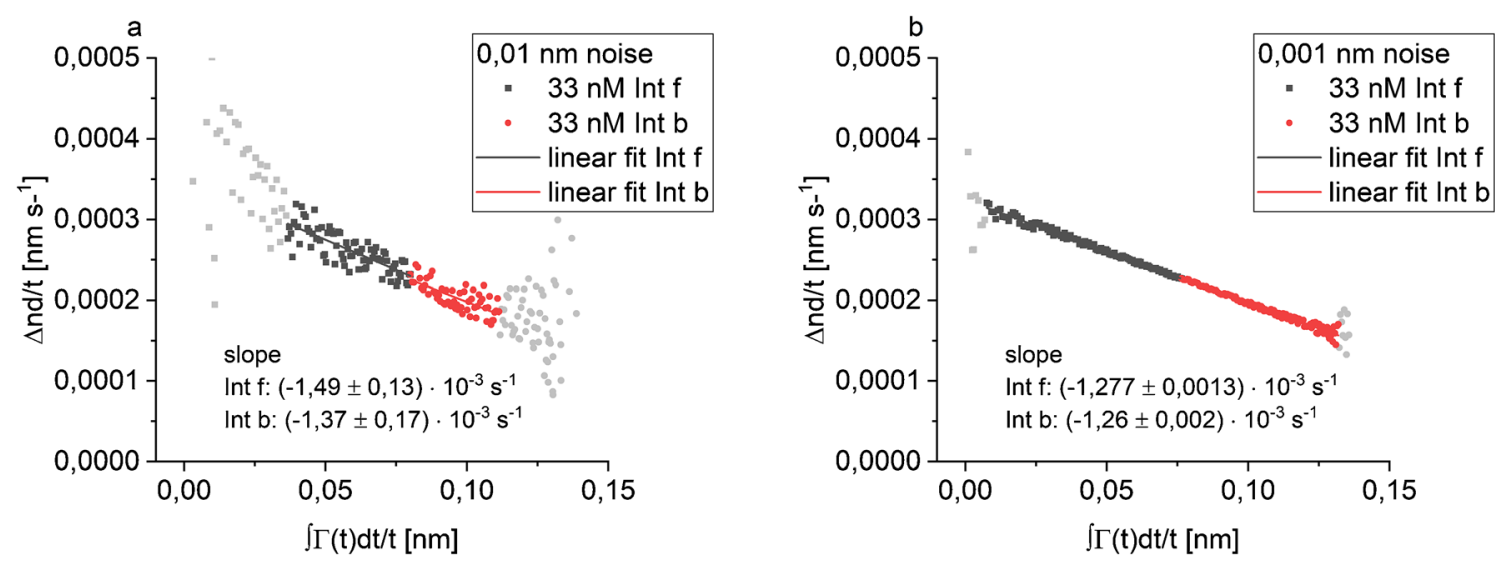

Fig. 1 Evaluation by integration of the entire association phase of simulated data for $33 \mathrm{nM}$, with $0.01 \mathrm{~nm}$ noise integrated from the beginning (Int f, dark grey) and from the end (Int b, red) with values deviating from linearity masked (light grey) (a) and for simulated data for $33 \mathrm{nM}$ with $0.001 \mathrm{~nm}$ noise (b)

\section{Results and discussion}

\section{Data evaluation of simulated data}

The evaluation of simulated data allows the comparison of the evaluation methods, if a perfect one-to-one interaction can be safely assumed. The simulated binding curves are shown in Fig. 2. It is obvious that data simulated with $0.001 \mathrm{~nm}$ noise (Fig. 2b) represents data comparable to good kinetic measurements because the noise is less than $1 / 100$ of the equilibrium surface load capacity of the smallest concentration, while data with $0.01 \mathrm{~nm}$ noise (Fig. 2a) would be considered in need of improvement of the experimental conditions.

\section{Rate constants}

For the simulated curves with $0.01 \mathrm{~nm}$ noise, $k_{\mathrm{a}}$ values calculated by all methods are shown in Table 1 . The calculated $k_{\mathrm{a}}$ values for the entire association deviate by less than $10 \%$ from the correct value for all methods as shown in Fig. 3a. In order to make a statement about the precision of the used methods, it

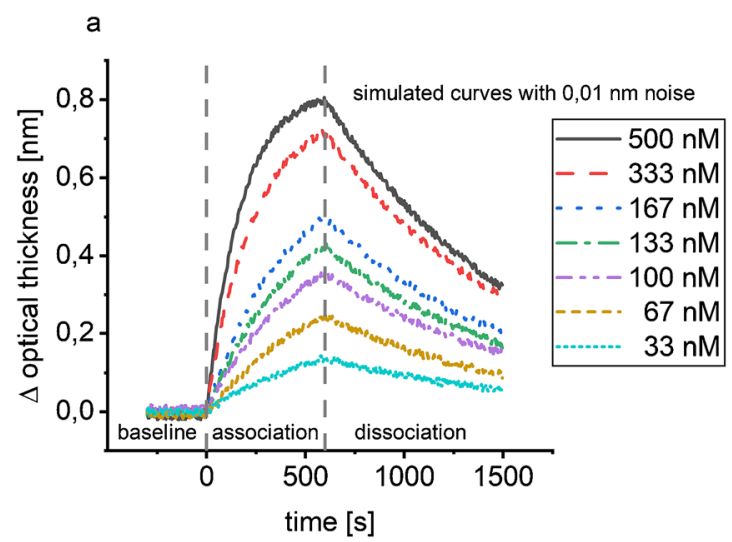

Fig. 2 Simulated binding curves with $0.01 \mathrm{~nm}$ (a) and $0.001 \mathrm{~nm}$ noise (b). Simulations were performed using the 1:1 (Langmuir) binding model in BIAevaluation 4.1.1 for seven different analyte concentrations with is considered which ones hit the correct value up to a deviation of $\pm 5 \%$. Both forward and backward integrations give $k_{\mathrm{a}}$ values deviating by less than $5 \%$ from the true value and the BIAevaluation methods as well. If only the first half of the association phase (300 s) is used for the calculation, all calculated $k_{\mathrm{a}}$ values deviate by less than $5 \%$ from the true value except for the backward integration. But if we take their standard errors of the fit into account, all used methods exceed a $5 \%$ deviation. (For BIAevaluation, the fitting region was not varied.) If the selection of the fitting area is based on the optical thickness and values are evaluated before reaching $0.5 \mathrm{~nm}$, the calculated $k_{\mathrm{a}}$ values with their standard errors of the fit exceed a $10 \%$ deviation of the true $k_{\mathrm{a}}$ value.

For the evaluation of the entire association phase, a $k_{\mathrm{a}}$ value deviating by less than $10 \%$ from the true value is obtained. As a deviation of $10 \%$ from the true value can be considered acceptable, it is concluded that all methods are in principle suitable for calculating $k_{\mathrm{a}}$, even if the signal is very noisy. The 5\% deviation criterion shows that forward and backward integrations of the entire association phase seem to be superior to the evaluation by exponential fit and derivative in the case

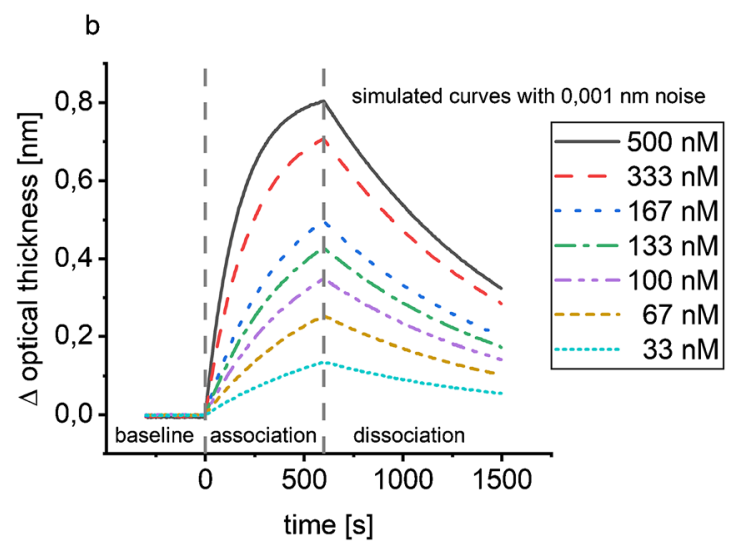

$300 \mathrm{~s}$ baseline, $600 \mathrm{~s}$ association, and $900 \mathrm{~s}$ dissociation; random noise with different amplitudes was added using Matlab 
Table $1 k_{\mathrm{a}}$ in $10^{4} \mathrm{M}^{-1} \mathrm{~s}^{-1}$ calculated with different methods for different fitting regions for simulated data with $0.01 \mathrm{~nm}$ noise. The $k_{\mathrm{a}}$ used for the simulation was $10^{4} \mathrm{M}^{-1} \mathrm{~s}^{-1}$

\begin{tabular}{lccccc}
\hline Method & $\begin{array}{l}\text { Entire association } \\
(0-600 \mathrm{~s})\end{array}$ & $\begin{array}{l}\text { 1st half of } \\
\text { association (time) }\end{array}$ & $\begin{array}{l}\Delta \mathrm{nd}< \\
0.5 \mathrm{~nm}\end{array}$ & $\begin{array}{l}\text { 1st half of } \\
\text { association }(\Delta \mathrm{nd})\end{array}$ & $\begin{array}{l}\text { 2nd half of } \\
\text { association }(\Delta \mathrm{nd})\end{array}$ \\
\hline Exp & $0.966 \pm 0.017$ & $0.95 \pm 0.03$ & $0.94 \pm 0.06$ & - & - \\
Der & $1.02 \pm 0.06$ & $0.97 \pm 0.12$ & $1.10 \pm 0.15$ & - & - \\
Int f & $0.99 \pm 0.03$ & $0.99 \pm 0.12$ & $0.98 \pm 0.11$ & $1.1 \pm 0.3$ & $0.96 \pm 0.15$ \\
Int b & $1.02 \pm 0.02$ & $0.94 \pm 0.09$ & $1.10 \pm 0.09$ & $1.1 \pm 0.2$ & $1.05 \pm 0.06$ \\
BIAsim & $0.988 \pm 0.003$ & - & - & - & - \\
BIAsep & $0.990 \pm 0.018$ & - & - & - & - \\
\hline
\end{tabular}

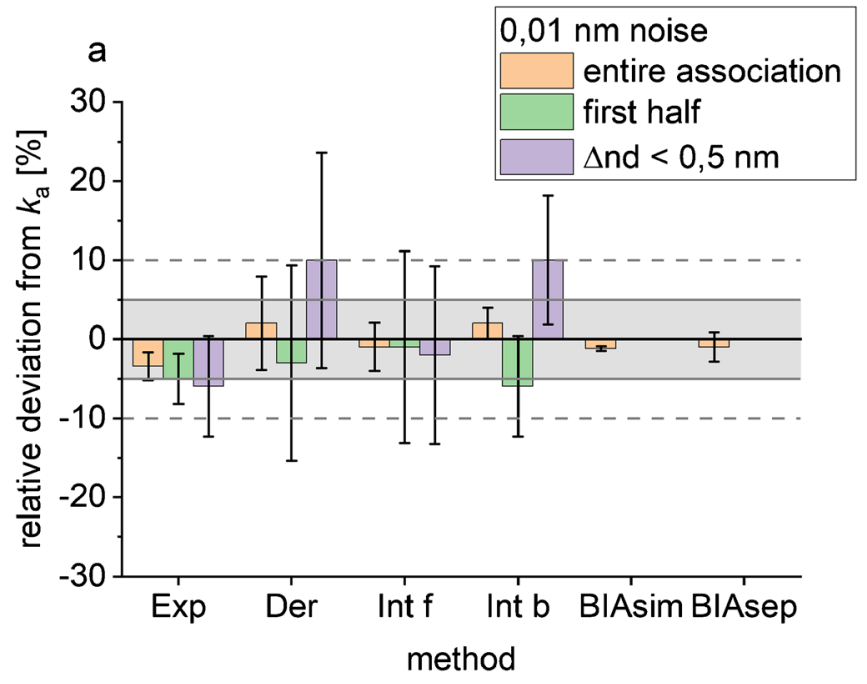

C

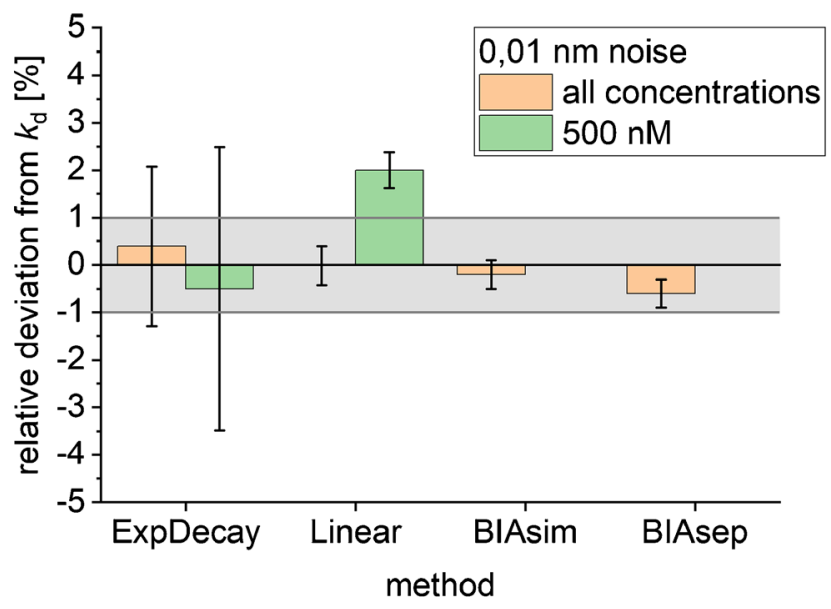

Fig. 3 Relative deviations of the rate constant from the true value and their relative standard error of the fit for different evaluation methods. The relative deviations from the true association rate constant were calculated for simulated data with $0.01 \mathrm{~nm}$ noise (a), with $0.001 \mathrm{~nm}$ noise (b) and relative deviation of the dissociation rate constants from the true value for simulated data with $0.01 \mathrm{~nm}$ noise (c), and with $0.001 \mathrm{~nm}$ noise (d). The association rate constant was calculated by $k_{\text {obs }}$ linearization after exponential fit (Exp) of the association phase, the derivative (Der), and forward and backward integration (Int $\mathrm{f}$, Int b) for different evaluation

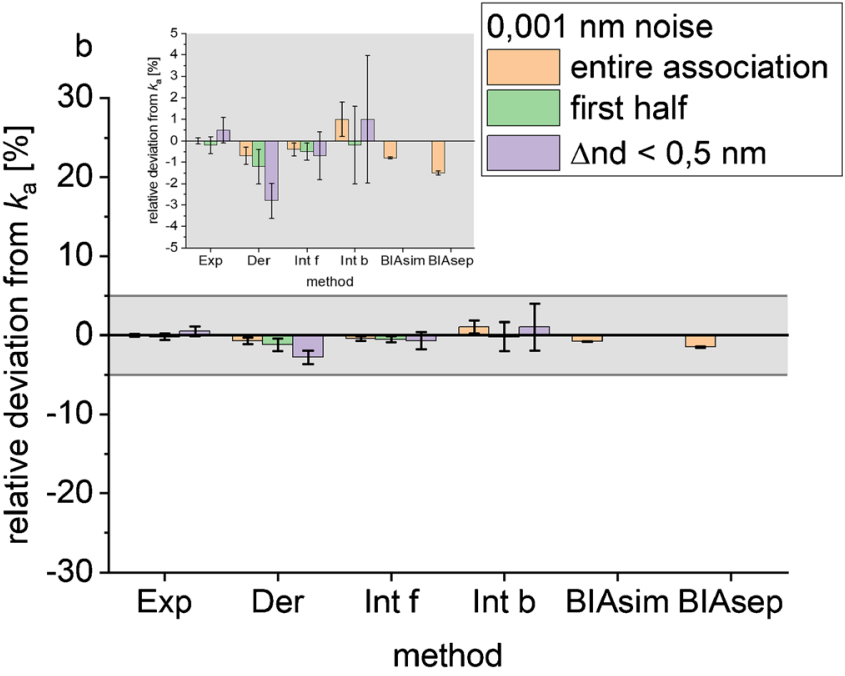

d

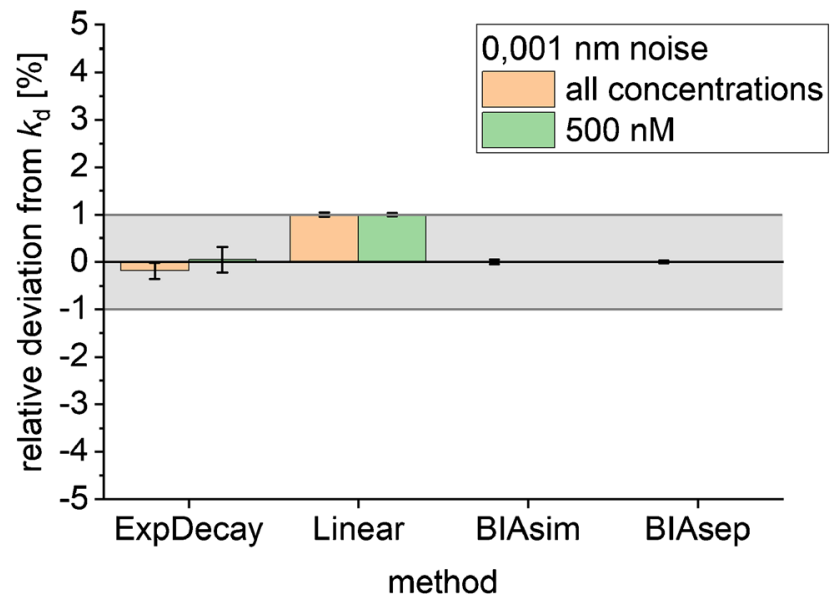

areas (entire association phase $(0-600 \mathrm{~s})$, first half $(0-300 \mathrm{~s})$, and from the beginning of the association phase until a $\Delta$ optical thickness of $0.5 \mathrm{~nm}$ is reached $(\Delta \mathrm{nd}<0.5 \mathrm{~nm})$. Global fit was used for calculating $k_{\mathrm{a}}$ and $k_{\mathrm{a}}$ by BIAevaluation with simultaneous $\mathrm{ka} / \mathrm{kd}$ (BIAsim) and separate $\mathrm{ka} / \mathrm{kd}$ (BIAsep) for all concentrations. Dissociation rate constants were calculated by an exponential decay (ExpDecay) function and a linearization (Linear) with a global fit for all concentrations and for $500 \mathrm{nM}$ 
of noisy signals. The reduction of the fitting region leads to larger deviations from the true value and larger standard errors as the amount of data points is reduced.

For less noisy signals $(0.001 \mathrm{~nm})$, association rate constants are calculated correctly (less than $5 \%$ deviation) with all methods as shown in Fig. 3b. Table 2 shows that the $k_{\mathrm{a}}$ value closest to the $k_{\mathrm{a}}$ value simulated is clearly obtained, if the entire association phase is evaluated with the (mono-)exponential fit.

The dissociation rate constant is calculated correctly for all methods. They show less than 5\% deviation from the true value for $0.01 \mathrm{~nm}$ noise (Fig. 3c), and less than $1 \%$ deviation for $0.001 \mathrm{~nm}$ noise (Fig. 3d). The closest values in the case of $0.01 \mathrm{~nm}$ noise are obtained with the linearization method performed for all concentrations and the BIAevaluation simultaneous $\mathrm{ka} / \mathrm{kd}$ fit. With less noise $(0.001 \mathrm{~nm})$, the best results are obtained with the BIAevaluation separate $\mathrm{ka} / \mathrm{kd}$ fit, BIAevaluation simultaneous $\mathrm{ka} / \mathrm{kd}$ fit, and fitting an exponential decay function to the largest concentration $500 \mathrm{nM}$.

\section{Derivative}

It is very interesting that the linearization methods show larger deviations especially when the part of the association evaluated is reduced. For the derivative, the noise largely affects the evaluation as the derivative increases the noise. If the derivative $\mathrm{d}(\Delta \mathrm{nd}) / \mathrm{d} t$ is plotted against the $\Delta$ optical thickness, this can result in problems with linear fit because the noise strongly affects the calculation of the observed rate constant, especially for small concentrations. Smaller concentrations are more affected by noise as the relative signal-to-noise ratio $(S / N)$ is smaller. When the derivative for noisy signals is plotted against the signal $(\mathrm{d}(\Delta \mathrm{nd}) / \mathrm{d} t$ vs. $\Delta$ nd plot $)$ to obtain $k_{\mathrm{obs}}$ from the slope, a decreasing slope will become imperceptible due to the noise (Fig. S2a). If the $S / N$ is very low, the slope is not significantly different from zero, showing that the evaluation by derivative is less suitable for noisy data. This is supported by the $k_{\text {obs }}$ linearization. When comparing the $k_{\mathrm{obs}}$ linearization for the different methods (Fig. 4a), it becomes obvious that the $k_{\mathrm{obs}}$ calculated by derivative shows the largest standard error of the fit, especially for small concentrations. These problems do not occur if the $S / N$ is large enough.

\section{Integration}

The integration method can better cope with noisy signals as integration reduces noise. Figure 1 shows the integration of the entire association phase for the lowest concentration $33 \mathrm{nM}$ for both noise levels. Unlike the derivative, no problem occurs with determining the slope for the data with $0.01 \mathrm{~nm}$ noise (Fig. 1a), if enough data points in the beginning of the integration are masked. The values calculated with forward and backward integration merge seamlessly as shown in Fig. 1b, which allows a nice control over a well-chosen evaluation area and cleanly determined slope.

However, especially for the first values of the integration, the noise still affects the calculated values even for the highest concentrations. The more the integration proceeds, the smoother the obtained curves. The deviation of the first values is less pronounced for the simulated data with less noise. But for strong noise, there is still a large deviation from the true values in the case of small concentrations. For the evaluation by integration, the $X$ values are the integrated curve divided by the time interval of the integration. As the integration procedure reduces noise, the $X$ values are not much affected by the noise in the signal. In contrast, the $Y$ values represent the difference between the optical thickness at the end of the integration and the optical thickness at the start, resulting in the noise showing its effect in the $Y$ values. When starting the integration from the end where this difference is lower because the curve flattens, the noise will affect the calculated values more, resulting in more values having to be masked to perform good linear fits. The effect of integration smoothing only shows after integration over a certain area. Therefore, the first values in the integration method should be masked for calculating $k_{\text {obs }}$.

The integration method offers a variety of possible starting points. Integration can be started at the beginning of the association phase, but also at the end or in the middle. Figure 5 shows that using all data points (evaluating the entire association phase) and starting in the beginning of the association
Table $2 k_{\mathrm{a}}$ in $10^{4} \mathrm{M}^{-1} \mathrm{~s}^{-1}$ calculated with different methods for different fitting regions for simulated data with $0.001 \mathrm{~nm}$ noise. The $k_{\mathrm{a}}$ used for the simulation was $10^{4} \mathrm{M}^{-1} \mathrm{~s}^{-1}$

\begin{tabular}{lccccc}
\hline Method & $\begin{array}{l}\text { Entire association } \\
(0-600 \mathrm{~s})\end{array}$ & $\begin{array}{l}\text { 1st half of } \\
\text { association (time) }\end{array}$ & $\Delta \mathrm{nd}<0.5 \mathrm{~nm}$ & $\begin{array}{l}\text { 1st half of } \\
\text { association }(\Delta \mathrm{nd})\end{array}$ & $\begin{array}{l}\text { 2nd half of } \\
\text { association }(\Delta \mathrm{nd})\end{array}$ \\
\hline Exp & $1.000 \pm 0.0014$ & $0.998 \pm 0.004$ & $1.005 \pm 0.006$ & - & - \\
Der & $0.993 \pm 0.004$ & $0.988 \pm 0.008$ & $0.972 \pm 0.008$ & - & - \\
Int f & $0.996 \pm 0.003$ & $0.995 \pm 0.004$ & $0.993 \pm 0.011$ & $0.99 \pm 0.02$ & $0.990 \pm 0.017$ \\
Int b & $1.01 \pm 0.008$ & $0.998 \pm 0.018$ & $1.01 \pm 0.03$ & $1.017 \pm 0.015$ & $1.016 \pm 0.014$ \\
BIAsim & $0.9920 \pm 0.0004$ & - & - & - & - \\
BIAsep & $0.9850 \pm 0.0008$ & - & - & - & - \\
\hline
\end{tabular}




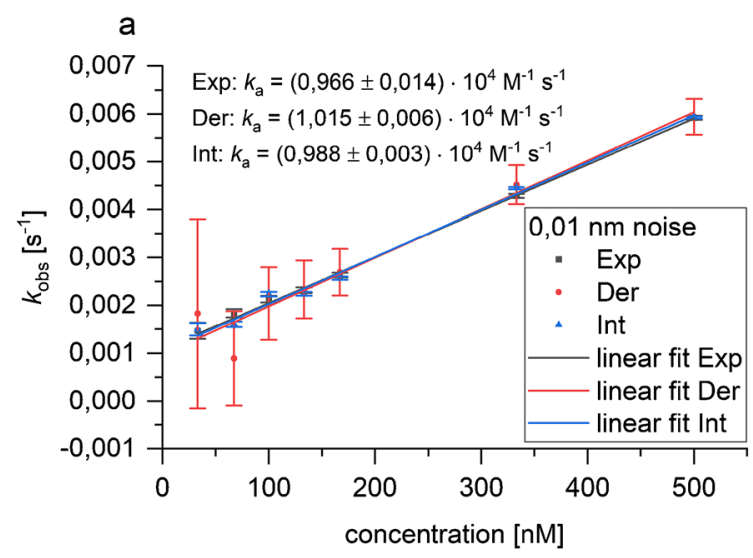

Fig. $4 k_{\text {obs }}$ linearization for simulated curves obtained with different evaluation methods for the entire association phase $(0-600 \mathrm{~s})$ with a $0.01 \mathrm{~nm}$ noise and $\mathbf{b} 0.001 \mathrm{~nm}$ noise. $k_{\mathrm{obs}}$ were calculated by

phase give the best results for both noise levels. Starting the evaluation in the middle where half of the final $\Delta$ nd is reached towards the end (Int $\mathrm{f}$ second half) gets a $k_{\mathrm{a}}$ value very close to the true value for very noisy signals (Fig. 5a). Starting in the middle and integrating towards the beginning of the association phase come very close for less noisy signals (Int $b$ first half in Fig. 5b).

Having the previous argumentation in mind that noise has less effect on the start of the integration where the signal increase is large, it makes sense to start the integration where the signal increase is larger. Thus, the integration should be started at the beginning or in the middle where half of the final $\Delta$ optical thickness is reached, but not at the end of the association phase. For real experiments, choosing the middle as the starting point would have the advantage that unwanted effects of the fluidics that might occur in the beginning of the association can be omitted.

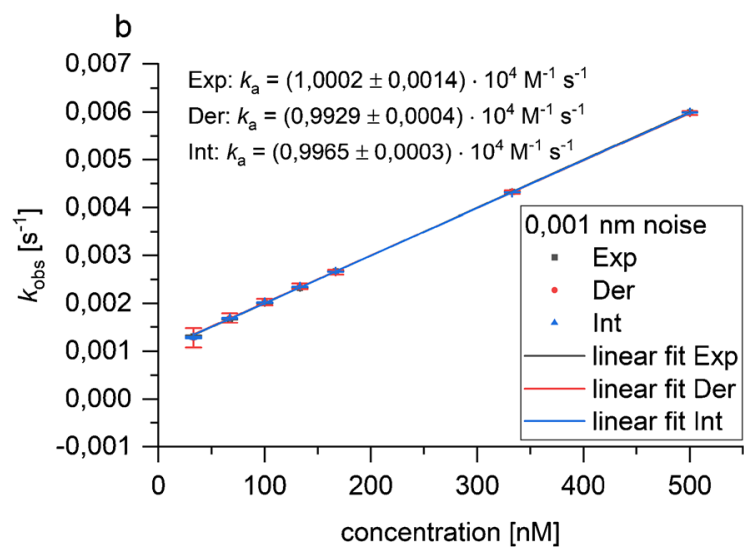

exponential fit (Exp), by derivative (Der), and by integration starting from the beginning of the association (Int). Error bars show the standard error of the fit. $k_{\mathrm{a}}$ is the slope

\section{Comparison of methods}

The evaluation of simulated binding curves shows that all considered methods are capable of calculating binding constants as they provide rate constants close enough to the true value. Table 3 shows a rating of the methods related to different aspects. All methods provide very good results, if the noise is small compared to the signal, which should be the case, if the experimental design is good. Only if the noise is very large compared to the signal do some methods lack accuracy. The comparison of very noisy and less noisy signal showed that the derivative leads to large errors, if the signal is very noisy, whereas the integration can better cope with noise. The integration can deal best with noisy data, because the integration method shows a smoothing effect. The BIAevaluation software also gives values very close to the true $k_{\mathrm{a}}$ value for noisy data.
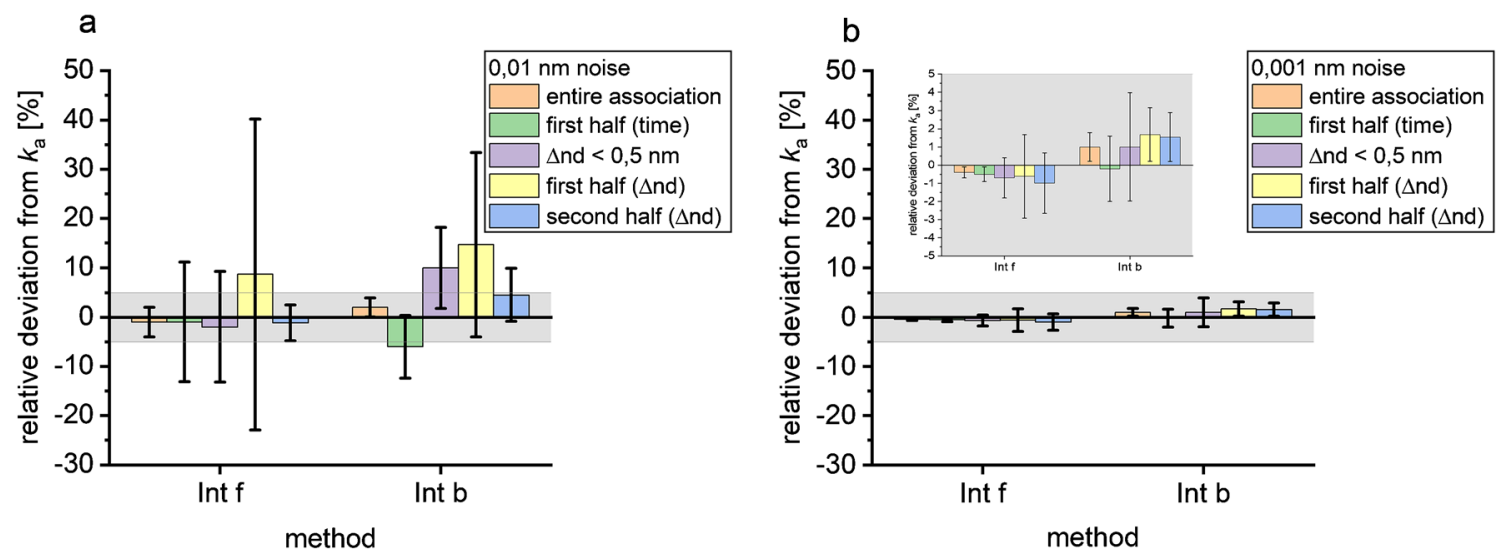

Fig. 5 Relative deviations of $k_{\mathrm{a}}$ calculated by the integration method for different evaluation ranges from the true value for data with $0.01 \mathrm{~nm}$ noise (a) and with $0.001 \mathrm{~nm}$ noise $(\mathbf{b})$. Error bars indicate the relative error of the fit 
Table 3 Rating of evaluation methods for the calculation of the association rate constant

\begin{tabular}{llllll}
\hline Method & Exp & Der & Int $\mathrm{f}$ & Int b & BIAevaluation \\
\hline Robustness against noise & - & - & ++ & + & ++ \\
Robustness against choice of evaluated region & + & - & + & - & Not examined \\
Independence of masking of data & + & + & - & - & + \\
\hline
\end{tabular}

The evaluation of different parts of the association phase shows that the best results are achieved when using the entire association phase. Using only parts of the association phase leads to a loss of information and thus to less precise results. In contrast, when evaluating experimental data, it can be advantageous to select the part of the association phase that matches the model. Exponential fit and forward integration can better deal with the reduction of the fitting area than derivative and backward integration. In general, the choice of the evaluated region is very important and as many data points as possible should be included.

In the case of the integration method, it was necessary to mask the first data points in the plot of $\Gamma(t) / t$ vs. $\int \Gamma(t) \mathrm{d} t / t$ in order to obtain good results whereas the other methods did not require additional data selection after the region for evaluation was chosen.

When residuals of the curve fitting of an experimental curve were added to a simulated binding curve, $k_{\text {obs }}$ was successfully determined by mono-exponential fit, derivative, and forward and backward integration (Fig. S3). The obtained $k_{\mathrm{obs}}$ values were sufficiently close to the simulated value deviating by less than $10 \%$ (Table S1). Thus, it is concluded that these evaluation methods should also be suitable for data with experimental noise. As the experimental data shown here can be compared to the simulated data with simulated noise of an amplitude of $0.001 \mathrm{~nm}$ with respect to their noise levels, similar results are expected when evaluating binding curves to which the residuals of experimental data are added.

\section{Data evaluation of measured data}

Example binding curves of the RIfS measurements are shown in Fig. 6. To check if one-to-one interaction is applicable, the natural logarithm of the derivative of the optical thickness of the association phase $\ln (\mathrm{d}(\Delta \mathrm{nd}) / \mathrm{d} t)$ can be plotted against time, which should be linear [32]. In Fig. S4, these checks are shown for an example $500 \mathrm{nM}$ measurement. The plot is linear for the association from 20 to $110 \mathrm{~s}$ for clone 202 and to $170 \mathrm{~s}$ for clone TU-11, respectively. For the dissociation phase, $\ln (\Gamma(0) / \Gamma(t))$ vs. time is plotted which deviates for both antibodies from a linear curve.

As the check for one-to-one interaction shows, one-to-one interaction cannot be assumed for the entire association phase. The derivative plotted vs. time is linear for a part of the association in the beginning, indicating that in this part using the model for pseudo-first-order reaction kinetics is possible. This simple model allows the comparison of the antibodies.

In the dissociation phase, a deviation from linearity is observed as shown in Fig. S4c and d, too. This shows that the criteria for a pseudo-first-order model are not completely met. Nevertheless, the deviation is rather small; the data only show a slight curvature.

This deviation from pseudo-first-order kinetics in the dissociation might be caused by the avidity of the antibody. If the antibody is bound to the surface with both paratopes, the dissociation will not follow pseudo-first-order kinetics and it might be possible that the antibody does not completely dissociate.

Even though the immobilization level was reduced to achieve low amounts of antigen on the surface and the flow rate was fast $(30 \mu \mathrm{l} / \mathrm{min})$, one-to-one interaction is not applicable. This result shows how difficult it is to design the experiment in the right way. A reason for the deviation could be the bivalency of the antibody or inhomogeneities. In this case, it was impossible to immobilize the antibody instead of the antigen because the binding of the antigen is too small to detect. Thus, avidity effects cannot be ruled out. Nevertheless, highquality binding curves were obtained and evaluated.

The plot of the derivative vs optical thickness shows that in the beginning of the association, the derivative can be approximated by a linear fit (Fig. S5a and c). For larger concentrations, it seems as if there is a kink in the derivative when an optical thickness of $0.5 \mathrm{~nm}$ for clone 202 and $0.6 \mathrm{~nm}$ for clone TU11 is reached. This linear part of the derivative was used for a linear fit to obtain $k_{\text {obs. }}$. In the $k_{\text {obs }}$ vs. $c$ plot shown in Fig. $\mathrm{S} 5 \mathrm{~b}$ and $\mathrm{d}$, the $k_{\mathrm{obs}}$ values for all concentrations lie on a straight line. Only the smallest concentration shows a larger deviation than the others for clone 202.

Evaluating the part of the association where the derivative shows linear behaviour with the model for pseudo-first-order kinetics is reasonable as for this part a one-to-one interaction can be assumed. The linearity of the $k_{\text {obs }}$ vs. $c$ plot confirms that this model can be used.

If only the part of the association phase where the derivative is linear is evaluated ( $\Delta \mathrm{nd}<0.5 / 0.6 \mathrm{~nm}$ ), the obtained values for $k_{\mathrm{a}}$ are significantly larger than if the entire association phase or the first half (time) is evaluated. When looking at the derivative of the large concentrations, it becomes obvious that evaluating more than the part up to the kink will lead to lower $k_{\mathrm{obs}}$ values for these concentrations, while the $k_{\mathrm{obs}}$ 

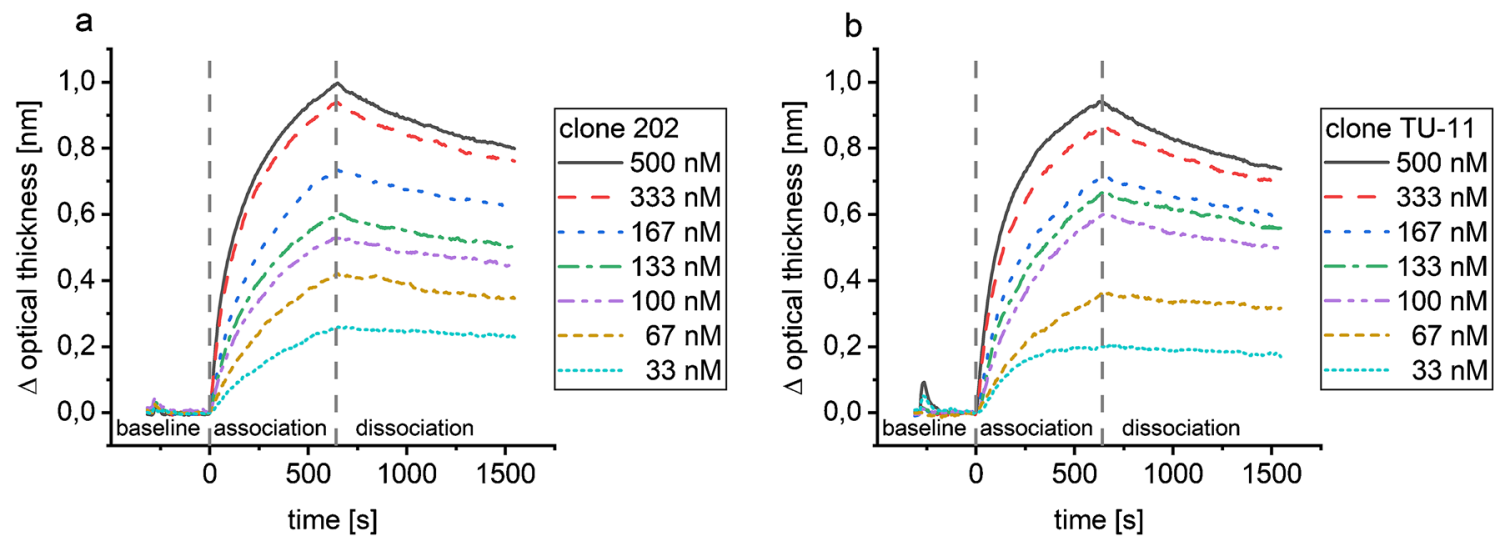

Fig. 6 Example RIfS measurements for each concentration of antibody clone 202 (a) and clone TU-11 (b). The baseline with PBS was followed by the injection of multiple analyte anti-amitriptyline antibody concentrations (association) and the dissociation of the antibody in PBS

values for the small concentrations will remain the same as these concentrations do not reach an optical thickness of 0.5 or $0.6 \mathrm{~nm}$. As only the larger $k_{\mathrm{obs}}$ values will decrease, this results in smaller slope when plotting $k_{\text {obs }}$ Vs. $c$, and consequently to a smaller $k_{\mathrm{a}}$ value. Fitting the entire association phase gives the average rate constant.

The kink in the derivative might be caused by an inhomogeneous surface, with some easily accessible surface sites to which the antibody can bind faster and some surface sites that are harder to reach and to which the antibody only binds when the easily accessible sites are occupied. This would mean that the antibody binds to different surface sites with different rate constants. Until a certain optical thickness is reached, the faster rate constant dominates.

The association rate constant was calculated with different methods for the part of the association phase showing linear behaviour in the derivative, and the obtained results for the $k_{\mathrm{a}}$ values are shown in Fig. 7. For both antibodies, the methods exponential fit, derivative, and forward integration provided the same $k_{\mathrm{a}}$ values, for clone 202 on average $2.4 \cdot 10^{4} \mathrm{M}^{-1} \mathrm{~s}^{-1}$

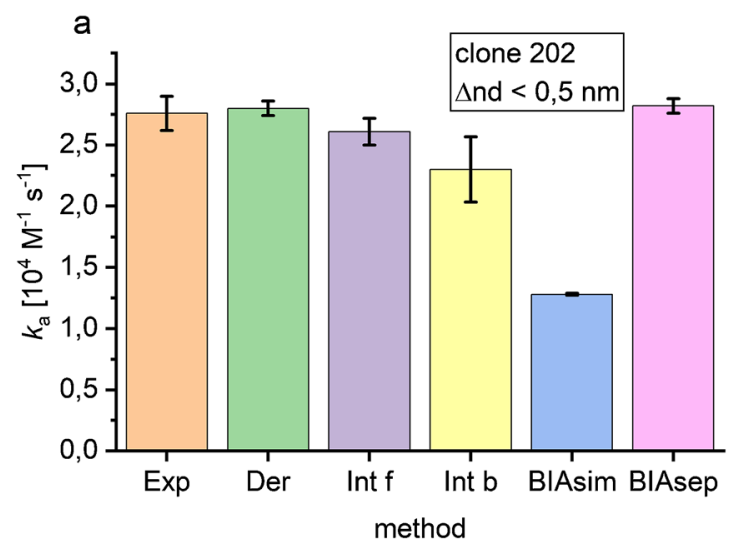

Fig. 7 Association rate constants calculated with different methods for antibody clone 202 (a) and clone TU-11 (b). The evaluated area was based on the linear behaviour in the derivative, i.e. until a $\Delta$ optical thickness of $0.5 \mathrm{~nm}$ was reached for clone 202 and $0.6 \mathrm{~nm}$ for clone and for clone TU-11 on average $1.9 \cdot 10^{4} \mathrm{M}^{-1} \mathrm{~s}^{-1}$. The backward integration and BIAevaluation simultaneous $\mathrm{ka} / \mathrm{kd}$ fit gave smaller $k_{\mathrm{a}}$ values, whereas the BIAevaluation separate $\mathrm{ka} / \mathrm{kd}$ fit gave larger $k_{\mathrm{a}}$ values. In both cases, the maximum $k_{\mathrm{a}}$ value, which was calculated with $2.8 \cdot 10^{4} \mathrm{M}^{-1} \mathrm{~s}^{-1}$ for clone 202 and $2.6 \cdot 10^{4} \mathrm{M}^{-1} \mathrm{~s}^{-1}$ for clone TU-11, were obtained by the BIAevaluation separate $\mathrm{ka} / \mathrm{kd}$ fit. All evaluation methods show that clone 202 has a larger association rate constant than clone TU-11, except the evaluation method BIAevaluation simultaneous $\mathrm{ka} / \mathrm{kd}$ fit. The minimum $k_{\mathrm{a}}$ value was for both antibodies calculated with the BIAevaluation simultaneous $\mathrm{ka} / \mathrm{kd}$ fit with $1.3 \cdot 10^{4} \mathrm{M}^{-1} \mathrm{~s}^{-1}$ for clone 202 and $1.5 \cdot 10^{4} \mathrm{M}^{-1} \mathrm{~s}^{-1}$ for clone TU-11.

As the evaluation methods (mono-)exponential fit, derivative, and integration all give similar rate constants, it can be concluded that the assumption of pseudo-first-order kinetics was justified. It is quite surprising that the two evaluation methods provided by the BIAevaluation software gave different results. The BIAevaluation software with its global fitting routines does not provide consistent data in this case. As all

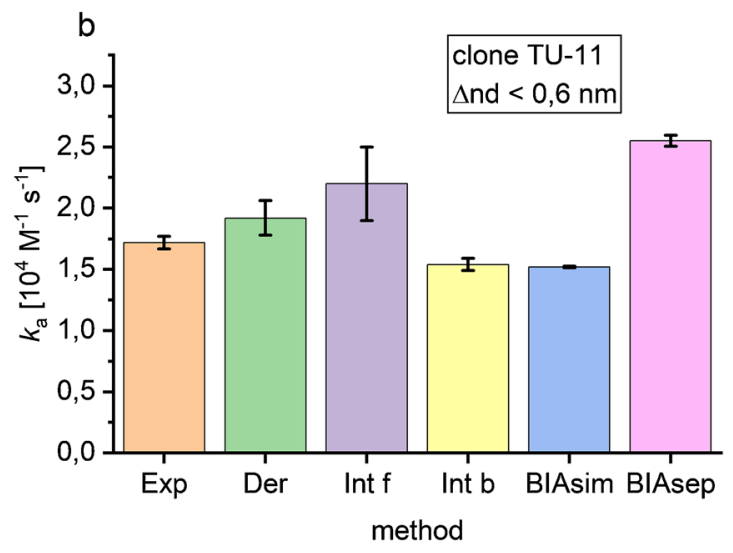

TU-11. Methods used were mono-exponential fit (Exp), derivative (Der), forward and backward integration (Int $\mathrm{f}$, Int b), simultaneous ka/ $\mathrm{kd}$ fit in BIAevaluation 4.1.1 (BIAsim), and separate $\mathrm{ka} / \mathrm{kd}$ fit in BIAevaluation (BIAsep). Error bars show the standard error of the fit 
methods except BIAevaluation simultaneous $\mathrm{ka} / \mathrm{kd}$ fit show a larger $k_{\mathrm{a}}$ value for antibody clone 202, it is concluded that this antibody associates faster.

To obtain the dissociation rate constants, all concentrations and the highest concentration were evaluated with four methods. The dissociation rate constants calculated by exponential fit and BIAevaluation with separate $\mathrm{ka} / \mathrm{kd}$ fit for $500 \mathrm{nM}$ are five to seven times larger than those calculated by other methods (Fig. 8). The average $k_{\mathrm{d}}$ value obtained with the exponential fit and the BIAevaluation separate $\mathrm{ka} / \mathrm{kd}$ fit for $500 \mathrm{nM}$ is $1.3 \cdot 10^{-3} \mathrm{~s}^{-1}$ for clone 202 and $1.4 \cdot 10^{-3} \mathrm{~s}^{-1}$ for clone TU-11, while the average $k_{\mathrm{d}}$ value obtained with the other methods is $2.3 \cdot 10^{-4} \mathrm{~s}^{-1}$ for clone 202 and $2.5 \cdot 10^{-4} \mathrm{~s}^{-1}$ for clone TU-11.

The difference between using the exponential decay function and the other methods is that the $y$-offset in the fitting function allows back bonding. This means that not all antibodies need to dissociate from the surface. If only the $500 \mathrm{nM}$ concentration is used for the BIAevaluation separate $\mathrm{ka} / \mathrm{kd}$ fit, the fit performed is basically the same as with the exponential decay function in Origin. BIAevaluation also has an offset implemented in its function which then allows the antibody not to fully dissociate. As the number of fitted parameters cannot exceed 31 in BIAevaluation, this offset was set to a global fit instead of a local fit as in Origin. If this global fit is performed for all concentrations, this fit effectively leads to the offset being very small as the best value for all concentrations has to be found. If it is locally fitted on the other hand, larger offsets are obtained for the higher concentrations. The smaller dissociation rate constants obtained with the other methods show the result that is obtained if the antibody has to dissociate completely. All in all, the $k_{\mathrm{d}}$ values obtained for the two antibodies show that they do not significantly differ in dissociation.

The calculation of rate constants for experimental data shows that different rate constants are obtained for different methods, but they are in a similar order of magnitude. If no strict one-to-one interaction can be assumed, but the data is still evaluated with this model, an average rate constant is obtained. The rate constant that more adequately describes the pseudo-first-order association rate constant is calculated by selecting only the linear part of the derivative as the fitting region. A linear slope for a part of the association phase indicates that during this time the interaction follows the one-toone model which allows the calculation of $k_{\mathrm{a}}$ for this part.

\section{Conclusion}

In summary, we show that different methods can be used for the evaluation of binding curves. The evaluation of simulated binding curves shows that different methods can be used to calculate the correct binding rate constants. The obtained $k_{\mathrm{a}}$ values when using linearization methods (derivation and integration) show larger deviations from the true values than other methods. Calculations by derivative and backward integration are less reliable since they are affected by noise. The values closest to the true $k_{\mathrm{a}}$ value are obtained using BIAevaluation or by forward integration of the entire association phase for very noisy data. For simulated data with noise in the range of real measurements, the values closest to the true $k_{\mathrm{a}}$ value are obtained using the fit with an exponential function or by evaluating with the forward integration followed by a $k_{\mathrm{obs}}$ linearization. Thus, the integration is strongly recommended.

For experimental data, association and dissociation rate constants can successfully be determined although no strict pseudo-first-order kinetic could be assured. Adapting the fitting region shows a change in the association rate constant. On the basis of the derivative, the part of the association where pseudo-first-order kinetics is followed is selected and evaluated. The rate constants calculated with different methods are of the same order of magnitude. Finally, the results here

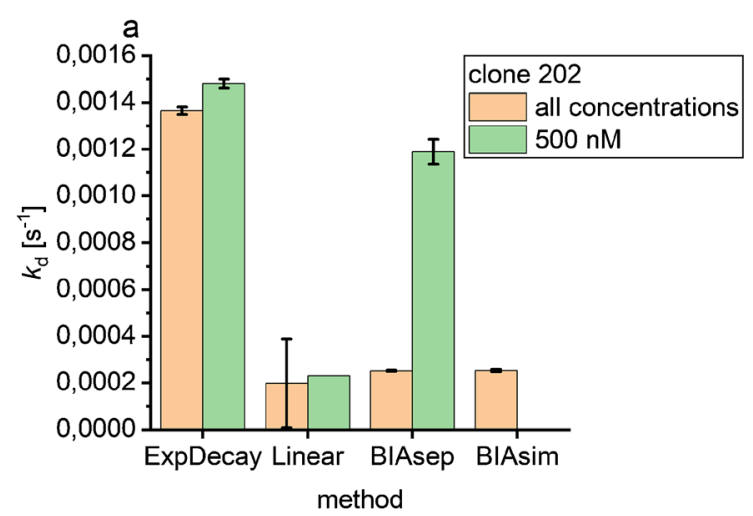

Fig. 8 Dissociation rate constants $k_{\mathrm{d}}$ calculated with different methods for antibody clone 202 (a) and antibody clone TU-11 (b). $k_{\mathrm{d}}$ was calculated with an exponential decay function (ExpDecay), a linearization method (Linear), the separate $\mathrm{ka} / \mathrm{kd}$ fit in BIAevaluation 4.1.1, and the

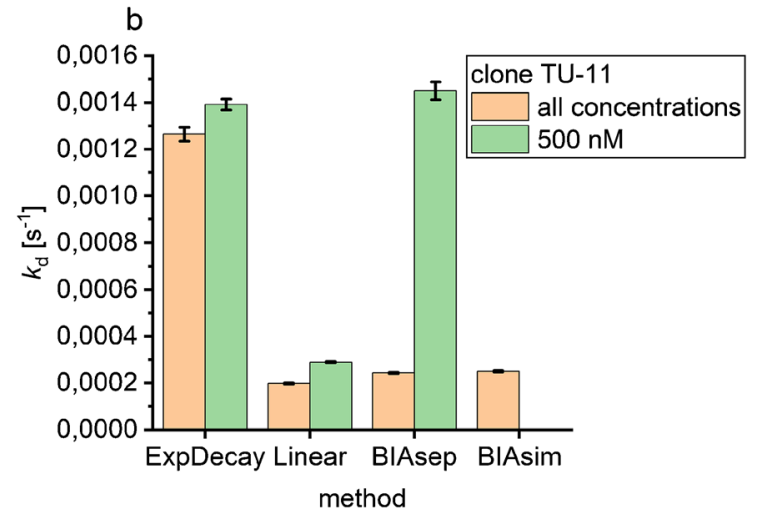

simultaneous $\mathrm{ka} / \mathrm{kd}$ fit in BIAevaluation for all concentrations and the highest concentration $500 \mathrm{nM}$. Error bars indicate the standard deviation of the fit 
suggest that looking at the derivative is very important as different rate constants become visible and it allows the selection of the part that follows pseudo-first-order kinetics. Evaluation with different methods of the same part of the association phase gives the same $k_{\mathrm{a}}$ value for exponential fit, derivative, and integration.

The results show that it is important not to rely on black box software, but instead to critically assess the data. We hope that this work will aid other researchers to generate more reliable data from BIA without having to rely on proprietary solutions.

Supplementary Information The online version contains supplementary material available at https://doi.org/10.1007/s00216-021-03623-x.

Acknowledgements We thank Florian Koelle from Cytiva Europe $\mathrm{GmbH}$ for providing the BIAevaluation 4.1.1 software and Christina Schanbacher for conducting RIfS measurements of antibody clone 202.

Availability of data and material The datasets generated and/or analysed during the current study are available from the corresponding author on reasonable request.

Author contribution All authors contributed to the study conception and design. Material preparation and data collection and analysis were performed by Monika Conrad. The first draft of the manuscript was written by Monika Conrad, and all authors commented on previous versions of the manuscript. All authors read and approved the final manuscript.

Funding Open Access funding enabled and organized by Projekt DEAL.

\section{Declarations}

Conflict of interest The authors declare no competing interests.

Open Access This article is licensed under a Creative Commons Attribution 4.0 International License, which permits use, sharing, adaptation, distribution and reproduction in any medium or format, as long as you give appropriate credit to the original author(s) and the source, provide a link to the Creative Commons licence, and indicate if changes were made. The images or other third party material in this article are included in the article's Creative Commons licence, unless indicated otherwise in a credit line to the material. If material is not included in the article's Creative Commons licence and your intended use is not permitted by statutory regulation or exceeds the permitted use, you will need to obtain permission directly from the copyright holder. To view a copy of this licence, visit http://creativecommons.org/licenses/by/4.0/.

\section{References}

1. Olaru A, Bala C, Jaffrezic-Renault N, Aboul-Enein HY. Surface plasmon resonance (SPR) biosensors in pharmaceutical analysis. Crit Rev Anal Chem. 2015. https://doi.org/10.1080/10408347. 2014.881250.

2. Rich RL, Myszka DG. Grading the commercial optical biosensor literature - class of 2008: 'The Mighty Binders'. J Mol Recognit. 2010. https://doi.org/10.1002/jmr.1004.
3. Rich RL, Myszka DG. Survey of the year 2005 commercial optical biosensor literature. J Mol Recognit. 2006. https://doi.org/10.1002/ jmr.808.

4. Schuck P, Minton AP. Kinetic analysis of biosensor data: elementary tests for self-consistency. Trends Biochem Sci. 1996. https:// doi.org/10.1016/s0968-0004(96)20025-8.

5. Myszka DG. Improving biosensor analysis. J Mol Recognit. 1999. https://doi.org/10.1002/(SICI)1099-1352(199909/10)12:5<279: AID-JMR473>3.0.CO;2-3.

6. Morton TA, Myszka DG. [13] Kinetic analysis of macromolecular interactions using surface plasmon resonance biosensors. In: Holt JM, Johnson ML, Ackers GK, editors. Energetics of biological macromolecules, vol. 295: Elsevier; 1998. pp. 268-294.

7. Katsamba PS, Navratilova I, Calderon-Cacia M, Fan L, Thornton K, Zhu M, Bos TV, Forte C, Friend D, Laird-Offringa I, Tavares G, Whatley J, Shi E, Widom A, Lindquist KC, Klakamp S, Drake A, Bohmann D, Roell M, et al. Kinetic analysis of a high-affinity antibody/antigen interaction performed by multiple Biacore users. Anal Biochem. 2006. https://doi.org/10.1016/j.ab.2006.01.034.

8. Liedberg B, Nylander C, Lunström I. Surface plasmon resonance for gas detection and biosensing. Sensors Actuators. 1983. https:// doi.org/10.1016/0250-6874(83)85036-7.

9. Karlsson R, Michaelsson A, Mattsson L. Kinetic analysis of monoclonal antibody-antigen interactions with a new biosensor based analytical system. J Immunol Methods. 1991. https://doi.org/10. 1016/0022-1759(91)90331-9.

10. Karlsson R, Mo JA, Holmdahl R. Binding of autoreactive mouse anti-type II collagen antibodies derived from the primary and the secondary immune response investigated with the biosensor technique. J Immunol Methods. 1995. https://doi.org/10.1016/00221759(95)00203-0.

11. Markgren P-O, Hämäläinen M, Danielson UH. Screening of compounds interacting with HIV-1 proteinase using optical biosensor technology. Anal Biochem. 1998. https://doi.org/10.1006/abio. 1998.2927.

12. Kampranis SC, Gormley NA, Tranter R, Orphanides G, Maxwell A. Probing the binding of coumarins and cyclothialidines to DNA gyrase. Biochem. 1999. https://doi.org/10.1021/bi982320p.

13. Lackmann M, Bucci T, Mann RJ, Kravets LA, Viney E, Smith F, Moritz RL, Carter W, Simpson RJ, Nicola NA, Mackwell K, Nice EC, Wilks AF, Boyd AW. Purification of a ligand for the EPH-like receptor HEK using a biosensor-based affinity detection approach. Proc Natl Acad Sci U S A. 1996. https://doi.org/10.1073/pnas.93.6. 2523.

14. Moll D, Prinz A, Gesellchen F, Drewianka S, Zimmermann B, Herberg FW. Biomolecular interaction analysis in functional proteomics. J Neural Transm. 2006. https://doi.org/10.1007/s00702006-0515-5.

15. Velazquez-Campoy A, Leavitt SA, Freire E. Characterization of protein-protein interactions by isothermal titration calorimetry. In: $\mathrm{Fu} \mathrm{H}$, editor. Protein-protein interactions. Methods and applications. Totowa, NJ: Humana Press; 2004. pp. 35-54.

16. Liedberg B, Nylander C, Lundström I. Biosensing with surface plasmon resonance - how it all started. Biosens Bioelectron. 1995. https://doi.org/10.1016/0956-5663(95)96965-2.

17. Damborský P, Švitel J, Katrlík J. Optical biosensors. Essays Biochem. 2016. https://doi.org/10.1042/EBC20150010.

18. Gauglitz G. Direct optical detection in bioanalysis: an update. Anal Bioanal Chem. 2010. https://doi.org/10.1007/s00216-010-3904-4.

19. Brandenburg A, Polzius R, Bier F, Bilitewski U, Wagner E. Direct observation of affinity reactions by reflected-mode operation of integrated optical grating coupler. Sensors Actuators B Chem. 1996. https://doi.org/10.1016/0925-4005(95)01747-J.

20. Schmitt H-M, Brecht A, Piehler J, Gauglitz G. An integrated system for optical biomolecular interaction analysis. Biosens Bioelectron. 1997. https://doi.org/10.1016/S0956-5663(97)00046-8. 
21. Gauglitz G. Critical assessment of relevant methods in the field of biosensors with direct optical detection based on fibers and waveguides using plasmonic, resonance, and interference effects. Anal Bioanal Chem. 2020. https://doi.org/10.1007/s00216-020-02581-0.

22. Biacore Insight Evaluation Software - Cytiva 2021. https://www. cytivalifesciences.com/en/us/shop/protein-analysis/spr-label-freeanalysis/software/biacore-insight-evaluation-software-p-23528. Accessed 1 Jun 2021.

23. TraceDrawer. 2021. https://tracedrawer.com/. .

24. Scrubber - BioLogic Software. 2017. http://www.biologic.com.au/ scrubber.html. .

25. Krämer SD, Wöhrle J, Rath C, Roth G. Anabel: an online tool for the real-time kinetic analysis of binding events. Bioinform Biol Insights. 2019. https://doi.org/10.1177/1177932218821383.

26. Svitel J, Balbo A, Mariuzza RA, Gonzales NR, Schuck P. Combined affinity and rate constant distributions of ligand populations from experimental surface binding kinetics and equilibria. Biophys J. 2003. https://doi.org/10.1016/S0006-3495(03)75132-7.

27. Gauglitz G. Analytical evaluation of sensor measurements. Anal Bioanal Chem. 2018. https://doi.org/10.1007/s00216-017-0624-z.

28. Chaiken IM, Rosé S, Karlsson R. Analysis of macromolecular interactions using immobilized ligands. Anal Biochem. 1992. https:// doi.org/10.1016/0003-2697(92)90329-6.

29. O'Shannessy DJ, Brigham-Burke M, Soneson KK, Hensley P, Brooks I. Determination of rate and equilibrium binding constants for macromolecular interactions using surface plasmon resonance: use of nonlinear least squares analysis methods. Anal Biochem. 1993. https://doi.org/10.1006/abio.1993.1355.

30. Glaser RW. Antigen-antibody binding and mass transport by convection and diffusion to a surface: a two-dimensional computer model of binding and dissociation kinetics. Anal Biochem. 1993. https://doi.org/10.1006/abio.1993.1399.

31. Schuck P. Kinetics of ligand binding to receptor immobilized in a polymer matrix, as detected with an evanescent wave biosensor. I A computer simulation of the influence of mass transport. Biophys J. 1996. https://doi.org/10.1016/S0006-3495(96)79681-9.

32. Karlsson R, Fält A. Experimental design for kinetic analysis of protein-protein interactions with surface plasmon resonance biosensors. J Immunol Methods. 1997. https://doi.org/10.1016/S00221759(96)00195-0.

33. Morton TA, Myszka DG, Chaiken IM. Interpreting complex binding kinetics from optical biosensors: a comparison of analysis by linearization, the integrated rate equation, and numerical integration. Anal Biochem. 1995. https://doi.org/10.1006/abio.1995.1268.

34. Mehne J, Markovic G, Pröll F, Schweizer N, Zorn S, Schreiber F, Gauglitz G. Characterisation of morphology of self-assembled PEG monolayers: a comparison of mixed and pure coatings optimised for biosensor applications. Anal Bioanal Chem. 2008. https://doi. org/10.1007/s00216-008-2066-0.

35. Krieg AK, Gauglitz G. Ultrasensitive label-free immunoassay for optical determination of amitriptyline and related tricyclic antidepressants in human serum. Anal Chem. 2015. https://doi.org/10. 1021/acs.analchem.5b01895.

36. Gauglitz G, Brecht A, Kraus G, Mahm W. Chemical and biochemical sensors based on interferometry at thin (multi-) layers. Sensors Actuators B Chem. 1993. https://doi.org/10.1016/0925-4005(93) 85234-2.

37. Bleher O, Ehni M, Gauglitz G. Label-free quantification of cystatin $\mathrm{C}$ as an improved marker for renal failure. Anal Bioanal Chem. 2012. https://doi.org/10.1007/s00216-011-5320-9.

38. Proll G, Kumpf M, Mehlmann M, Tschmelak J, Griffith H, Abuknesha R, Gauglitz G. Monitoring an antibody affinity chromatography with a label-free optical biosensor technique. J Immunol Methods. 2004. https://doi.org/10.1016/j.jim.2004.05. 008 .

Publisher's note Springer Nature remains neutral with regard to jurisdictional claims in published maps and institutional affiliations. 